\title{
Toward Pan-Slavic NLP: Some Experiments with Language Adaptation
}

\author{
Serge Sharoff \\ Centre for Translation Studies \\ University of Leeds, Leeds, UK \\ s.lastnamed leeds.ac.uk
}

\section{Introduction}

There is great variation in the amount of NLP resources available for Slavic languages. For example, the Universal Dependency treebank (Nivre et al., 2016) has about $2 \mathrm{MW}$ of training resources for Czech, more than $1 \mathrm{MW}$ for Russian, while only 950 words for Ukrainian and nothing for Belorussian, Bosnian or Macedonian. Similarly, the Autodesk Machine Translation dataset only covers three Slavic languages (Czech, Polish and Russian). In this talk I present a general approach, which can be called Language Adaptation, similarly to Domain Adaptation. In this approach, a model for a particular language processing task is built by lexical transfer of cognate words and by learning a new feature representation for a lesser-resourced (recipient) language starting from a better-resourced (donor) language. More specifically, I demonstrate how language adaptation works in such training scenarios as Translation Quality Estimation, Part-of-Speech tagging and Named Entity Recognition.

\section{Transfer of Feature Representation}

Machine Learning algorithms are limited by the availability of training data. This problem is often addressed by developing algorithms to transfer NLP models across different domains, for example, an opinion mining model trained on IMDb can be transferred to the domain of hotel reviews (Søgaard, 2013). In a similar way, we can assume that a model trained in a donor language can be transferred to a recipient language relying on the fact that both languages come from the same language family.

One of the observations for transferring models across languages is that while the general assumption of similarity holds, the individual features exhibit a slightly different distribution. For example,

\begin{tabular}{|c|c|c|c|}
\hline \multirow{2}{*}{\multicolumn{2}{|c|}{ Upper baseline (ru) }} & MAE & 0.18 \\
\hline & & RSME & 0.27 \\
\hline & & Pearson & 0.47 \\
\hline en-ru & $\rightarrow$ & en-cs & en-pl \\
\hline \multirow{3}{*}{ STL } & MAE & 0.19 & 0.19 \\
\hline & RMSE & 0.25 & 0.25 \\
\hline & Pearson & 0.41 & 0.46 \\
\hline \multirow{3}{*}{$\begin{array}{l}\text { Baseline } \\
\text { Train: ru } \\
\text { Test: } x x\end{array}$} & MAE & 0.20 & 0.21 \\
\hline & RMSE & 0.26 & 0.27 \\
\hline & Pearson & 0.32 & 0.33 \\
\hline
\end{tabular}

Table 1: STL for MT Quality Estimation.

in the task of estimating MT quality without reference translations, good MT examples are similar in the feature space describing translation into two related languages, but the exact feature values, such as the Language Model values or the phrase table sizes differ. One way of transferring the feature spaces is via Self-Taught Learning (STL), in which an autoencoder learns to reduce the dimensions of unlabelled datasets for the two domains. Then the available training set in one domain is transformed using the autoencoder, so that a new prediction model can be equally successful in the source domain and in the new target domain (Raina et al., 2007). As shown in (Rios and Sharoff, 2016), an application of this transformation to predicting the amount of Post-Editing needed to improve raw MT output can produce models which almost reach the accuracy of the original prediction model (Table 1).

\section{Transfer of Lexica}

Linguistic models can be also transferred through re-using grammatical models trained in a donor language with substitution of the lexicons from a recipient language. For example, a POS tagger can use the transition probabilities from the donor, 
while the lexical emission probabilities can come from the recipient (Feldman et al., 2006; Reddy and Sharoff, 2011).

Similarly, a traditional MT engine for translation from Ukrainian into English and German can be surpassed by a crude MT pipeline consisting of a direct word-for-word transfer model from Ukrainian into Russian followed by a better resourced model translating from Russian into English and German (Babych et al., 2007). The reason for the success of the pipeline is that the OutOf-Vocabulary rate is reduced primarily because of the better coverage of the donor lexicon.

Automatic induction of translation lexica between related languages is easier than in the more general case, since in addition to the similarity of the embedding vectors, they often have very similar forms. A reliable lexicon can be produced by combining detection of cognate forms via Levenshtein distance with assessment of semantic similarity via bilingual word embeddings even in the absence of parallel corpora (Upadhyay et al., 2016). One of the problems in transferring the lexica concerns Multi-Word Expressions (MWEs), which tend to differ even for closely related languages. In particular, this concerns fixedform MWEs without a defined grammatical structure, such as by and large or of course in English. Such MWEs need to be detected individually in each language and linked to a grammatical model in a donor language via a distributional measure of their similarity to single-word expressions, e.g., generally or definitely in the examples above (Riedl and Biemann, 2015).

In my talk I have also demonstrated an end-toend example for transferring feature spaces and lexicons by developing a Named Entity Recognition tagger, which starts with resources available for Slovene and transfers the features derived from a CRF model (Lafferty et al., 2001; Benikova et al., ) to other Slavic languages.

\section{References}

Bogdan Babych, Anthony Hartley, and Serge Sharoff. 2007. Translating from under-resourced languages: comparing direct transfer against pivot translation. In Proceedings of MT Summit XI, pages 412-418, Copenhagen.

Darina Benikova, Seid Muhie Yimam, Prabhakaran Santhanam, and Chris Biemann. GermaNER: Free open German named entity recognition tool. In Pro- ceedings of the International Conference of the German Society for Computational Linguistics and Language Technology (GSCL 2015), pages 31-38, University of Duisburg-Essen, Germany.

Anna Feldman, Jirka Hana, and Chris Brew. 2006. A cross-language approach to rapid creation of new morpho-syntactically annotated resources. In Proceedings of the 5th International Conference on Language Resources and Evaluation (LREC 2006), pages 549-554, Genoa, Italy.

John Lafferty, Andrew McCallum, and Fernando Pereira. 2001. Conditional random fields: Probabilistic models for segmenting and labeling sequence data. In Proceedings of the eighteenth international conference on machine learning, ICML, volume 1, pages 282-289.

Joakim Nivre, Marie-Catherine de Marneffe, Filip Ginter, Yoav Goldberg, Jan Hajic, Christopher D. Manning, Ryan McDonald, Slav Petrov, Sampo Pyysalo, Natalia Silveira, Reut Tsarfaty, and Daniel Zeman. 2016. Universal Dependencies v1: A multilingual treebank collection. In Proceedings of the 10th International Conference on Language Resources and Evaluation (LREC 2016), pages 1659-1666.

Rajat Raina, Alexis Battle, Honglak Lee, Benjamin Packer, and Andrew Y. Ng. 2007. Self-taught learning: Transfer learning from unlabeled data. In Proceedings of the 24th international conference on Machine learning, pages 759-766. ACM.

Siva Reddy and Serge Sharoff. 2011. Cross language POS taggers (and other tools) for Indian languages: An experiment with Kannada using Telugu resources. In Proceedings of the Fifth International Workshop On Cross Lingual Information Access, pages 11-19.

Martin Riedl and Chris Biemann. 2015. A single word is not enough: Ranking multiword expressions using distributional semantics. In Proceedings of the 2015 Conference on Empirical Methods in Natural Language Processing, pages 2430-2440, Lisboa, Portugal.

Miguel Rios and Serge Sharoff. 2016. Language adaptation for extending post-editing estimates for closely related languages. The Prague Bulletin of Mathematical Linguistics, 106(1):181-192.

Anders Søgaard. 2013. Semi-Supervised Learning and Domain Adaptation in Natural Language Processing. Synthesis Lectures on Human Language Technologies. Morgan \& Claypool Publishers.

Shyam Upadhyay, Manaal Faruqui, Chris Dyer, and Dan Roth. 2016. Cross-lingual models of word embeddings: An empirical comparison. In Proceedings of the 54th Annual Meeting of the Association for Computational Linguistics, pages 16611670, Berlin, Germany. 